\title{
Are there any guarantees with the warranty period for normal stress SPECT myocardial perfusion imaging?
}

\author{
Gary R. Small, MBChB, PhD, ${ }^{a}$ and Terrence D. Ruddy, $M^{a}$ \\ a Division of Cardiology, University of Ottawa Heart Institute, Ottawa, ON, Canada
}

Received May 31, 2018; accepted Jun 1, 2018

doi: 10.1007/s12350-018-1334-4

\section{See related article, pp. 534-541}

The Oxford English dictionary defines a warranty as a written guarantee from the manufacturer promising to repair or replace a product within a specified period of time. In this issue of the journal Acampa et al. report the warranty period of a normal stress myocardial perfusion imaging (MPI) in hypertensive patients. ${ }^{1}$ The authors determined the warranty period as the length of time that a patient would remain at low risk, defined as a $<3 \%$ cumulative event rate for cardiac death or non-fatal myocardial infarction (MI) based on 6 years of follow up. Although a reassuring term, a warranty period does belie the sense of uncertainty in clinical medicine and may foster an indifferent approach to lifestyle and therapeutic compliance. If interpreted appropriately, a warranty period can nevertheless provide useful risk stratification data to physicians and their patients.

\section{WARRANTY PERIODS FOR A NORMAL SPECT- MPI}

The concept of a low-risk warranty period following a normal SPECT-MPI was introduced by Hachamovitch et al. in $2003 .^{2}$ It was based on the observation that following a normal MPI, patients may experience different cardiac event rates depending on their clinical

Reprint requests: Terrence D. Ruddy, MD, Division of Cardiology, University of Ottawa Heart Institute, Ottawa, ON, Canada; truddy@ottawaheart.ca

J Nucl Cardiol 2020;27:542-6.

$1071-3581 / \$ 34.00$

Copyright (c) 2018 American Society of Nuclear Cardiology. risk factors. Although multivariate analysis can discover which risk factors are associated with increased event rates following a normal study, in order to calculate a warranty period, analysis is required that allows for temporal changes in the influence of risks. Survival analysis has been used to model the relationship between risk factors and the warranty period. An ideal algorithm would not presume that event rates would remain static over time or that the influence of risks would remain the same. Typically, survival analysis is undertaken using Cox proportional hazards model which can accommodate covariables to control for age, gender, and other cardiovascular risks. However, Cox proportional hazards analysis assumes both a constant survival rate and a constant effect of the "hazards" and does not make assumptions to account for the possible acceleration of events with time as might be expected with atherosclerosis. Hachamovitch, Acampa, and others have therefore used parametric regression modeling which includes an assumption that the predictors or risks themselves are in direct relationship with survival. ${ }^{1-4}$ Hypertension, age, and diabetes are all risks expected to accelerate the rate of increase in events. Acampa et al. used the Weibull model for survival analysis which has been used in atherosclerosis survival modeling since the assumptions of this model have been demonstrated to be the best fit in comparison to other parametric regression models (exponential, log-normal, log-logistic). ${ }^{1,2}$ In calculating a warranty period using parametric survival analysis, the period is derived from the observed rate of survival decrease (survival function), the predicted event rate based on the presence of risks (hazard function), and the expected relationship between the presence of hazards and survival decline (accelerated failure time). Finally to determine the warranty period, a decision is required to set the threshold of event rates beyond which a patient is no longer considered low risk. 


\section{CHOICE OF AN EVENT RATE FOR LOW RISK OF CARDIAC DEATH OR NON-FATAL MI}

Acampa et al. chose a cumulative event rate threshold of $3 \%$ as this was the rate of events in their low-risk cohort at 60 months (event rate with normal perfusion with exercise stress in hypertensives $<60$ years of age). ${ }^{1}$ In patients $\geq 60$ years of age, the low-risk warranty lasted 23 months, beyond which time the rates of cardiac death or non-fatal MI were $\geq 3 \%$. Of note, the annual event rates for hypertensive patients $(1.3 \%)$ were almost 3 fold greater than for normotensives $(0.4 \%)$ $(P=0.004)$. Thus the interpretation of a low-risk warranty period for hypertensives requires an understanding that hypertensive patients already have an increased risk for cardiac events. It could be argued that a true low-risk warranty period for those with hypertension does not exist as their event rates were always greater than $0.4 \%$ per annum.

In a previous study, Acampa et al. had considered the warranty period of a normal MPI in diabetic population of 260 patients and propensity matched controls. ${ }^{3}$ A cumulative event rate threshold of $>3 \%$ was chosen in this cohort as this was the event rate in the non-diabetics with normal perfusion imaging. An event rate of $>3 \%$ was achieved after 145 months in nondiabetics, whilst in diabetics this low-risk period lasted 42 months with normal MPI.

Another European research group has also considered the concept of a warranty period following normal SPECT. ${ }^{4}$ This group considered low risk to be $<1 \%$ annual events (cardiovascular mortality and non-fatal MI). In 2922 consecutive patients with normal MPI, they found that the stressor, exercise versus dipyridamole, influenced the warranty period. They also determined that age, gender, diabetes, and known coronary artery disease (CAD) influenced the length of time a patient was at low risk. Patients with exercise stress, MPI had a warranty period of 43.4 months versus 17.8 months for dipyridamole stress MPI. Non-diabetics had a warranty period of 46 months and diabetics 36 months. Those patients $<65$ years of age remained at low risk $(<1 \%)$ for 60 months; whereas those $\geq 65$ years experienced low-risk status for 24 months. Perhaps in light of these findings the true warranty period $(<1 \%$ per year) for a normal MPI in the presence of cardiovascular risk factors can be quite short but varies considerably.

Hachamovitch et al. described the warranty period following a normal MPI using thresholds of cumulative event rates of $0.5,1,1.5$, and $2 \%$ in 7,376 consecutive patients. ${ }^{2}$ Events were defined as cardiac mortality or non-fatal MI and follow up was a mean of $665 \pm 200$ days. Age, diabetes, male gender, prior $\mathrm{CAD}$, and

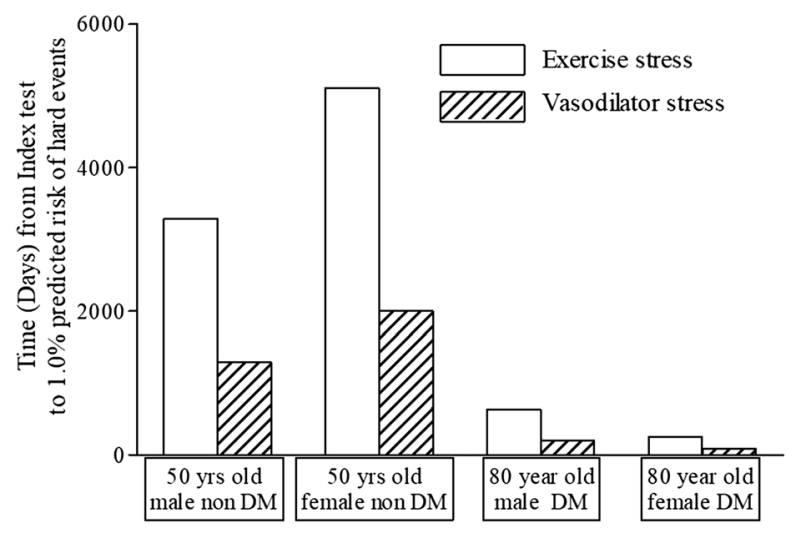

Figure 1. Varying warranty periods determined using survival analysis in patients without a known history of CAD undergoing exercise or vasodilator stress SPECT myocardial perfusion imaging showing the effects of stress type, age and gender. Hard events were classified as cardiac death and nonfatal myocardial infarction. ${ }^{2}$.

adenosine-stress were associated with shorter time to events (Figure 1).

Across these 4 studies of patients with a normal MPI, clinical risks provide incremental prognostic data to predict survival free duration. ${ }^{1-4}$ Age, diabetes, and hypertension were shown to be predictive of events even in the presence of a normal study. Consistently, event incidence increased from initial low levels (the warranty period) at varying rates of acceleration depending upon the constellation of cardiovascular risks.

\section{DOES ATHEROSCLEROSIS BEGET ATHEROSCLEROSIS?}

Can the rates of accelerated decline be explained by observational studies examining the natural progression of atherosclerosis? Long term data cataloging the progression of coronary atheroma are available from CT coronary calcification (CAC) data and from the MESA study (Multi-Ethnic Study of Atherosclerosis)., ${ }^{5,6}$ Min et al. considered the warranty period for a normal CAC-CT scan (Agatston score $=0$ ) in 422 patients with a mean age of 49 years who underwent annual CAC-CT scanning for 5 years. ${ }^{6}$ About $25 \%$ of patients developed an Agatston score $>0$. The development of CAC was non-linear and followed a low incidence before 4 years and accelerated in year 5. Conversion from $\mathrm{CAC}=0$ to a CAC $>0$ occurred in $0.5 \%, 1.2 \%, 5.7 \%, 6.2 \%$, and $11.6 \%$ of patients for years $1-5$ respectively. Traditional $\mathrm{CV}$ risk factors including, diabetes, age, hypertension, and smoking were independently associated with conversion to $\mathrm{CAC}>0$. Min also considered a parallel group of 621 patients with CAC $>0$ on the initial CAC-CT. In this cohort of patients, the effects of $\mathrm{CV}$ risks on the 
progression of CAC were recorded on annual CT studies and CAC progression was associated with hyperlipidemia, smoking, male gender, and baseline CAC score. Yet, the only independent predictor for the risk of CAC progression using a multivariate survival analysis was CAC itself. Diabetes, smoking, or hypertension did not significantly increase the hazard of progression once CAC was included in the model. The absence of an effect of clinical CAD risks may have reflected the powerful predictive nature of CAC or the short period of follow up (1.9 \pm 1.1 years) in this cohort.

The MESA study performed repeat CAC-CT scanning in 2948 patients with $\mathrm{CAC}=0$ at baseline. They found $16 \%$ developed a CAC $>0$ over 2.4 years of follow up. ${ }^{5}$ The shorter period of follow up precluded a long term survival analysis to calculate a warranty period. They were able to determine the effects of ethnicity/race on CAC progression with white males experiencing a $10.3 \%$ conversion versus $4.4 \%$ in Chinese men (age adjusted $P<0.001$ ). Following backward selection modeling incident CAC was associated with aging, ethnicity/race, body mass index, use of antihypertensive medications, LDL cholesterol, triglycerides, use of lipid lowering medications, diabetes, family history of CAD, and serum creatinine. MESA also included 2808 patients with CAC $>0$ at baseline. Progression of CAC was associated with length of follow up, age at baseline, male gender, white ethnicity, higher BMI, hypertension, family history of CAD, and diabetes. In the MESA population, diabetes was the strongest risk factor for CAC progression. ${ }^{5}$

From these and other studies cataloging progression of CAC as marker of coronary atherosclerosis, we can confirm the influence of CAD risk factors to contribute to the progression of atherosclerosis. ${ }^{7}$ Furthermore, the presence of atherosclerosis itself appears to be an important determinant for the development of greater degrees of atherosclerotic burden as indicated by the CAC score.

\section{MPI SPECT AND NON-OBSTRUCTIVE ATHEROSCLEROSIS}

Can nuclear cardiology imaging data also document progressive atherosclerosis in the absence of obstructive CAD? With SPECT-CT cameras with $\geq 16$ slice CTs, the presence of CAC can be determined using the attenuation correction CT images. ${ }^{8}$ Using the RETROSPECT-data base (Registry for the evaluation of the prognostic value of a novel integrated imaging approach combining single photon emission computed tomography with coronary calcification imaging), Ghadri et al. studied 462 consecutive patients with stress only ${ }^{99 \mathrm{~m}} \mathrm{Tc}$ tetrofosmin SPECT/ CT imaging. . Mean age was
$63.4 \pm 11.3$ years and follow up was $34.5 \pm 13$ months with an endpoint of composite MACE (death, non-fatal MI, unstable angina and revascularization). Normal perfusion imaging with a CAC $>0$ was seen in 262 patients. These cases comprised the majority of a group of 274 cases where the findings of the SPECT and CAC were seen to be unmatched (Either abnormal perfusion with no CAC or vice versa). This group had a $16 \%$ MACE rate over the follow up period, in comparison to $36 \%$ and $0 \%$ respectively in the matched (perfusion and $\mathrm{CAC}$ abnormal) and normal (MPI and $\mathrm{CAC}=0$ ) groups. Although no warranty period was calculated in this study, the additive data from coronary atherosclerosis imaging with $\mathrm{CT}$ proved powerfully predictive for MACE. In the absence of CAC data, the majority of patients in the mismatch group would have been given a normal scan diagnosis and their prognosis was not low risk.

SPECT myocardial blood flow measurement might also be used to detect coronary atherosclerosis. Although not in widespread clinical use, SPECT-MPI blood flow quantification has been validated and may provide diagnostic and prognostic information which is incremental to relative perfusion imaging (Figure 2). ${ }^{10,11}$ Gould et al. proposed that stress PET myocardial blood flow might be considered a continuum between normal through impaired - non obstructive atherosclerosis to obstructive coronary artery disease. ${ }^{12}$ SPECT myocardial blood flow measurement may provide a similar schema. In cases of normal relative perfusion imaging, abnormal or reduced blood flow would be expected to have prognostic significance based on PET data. ${ }^{13}$ A warranty period for normal perfusion studies (based on the event threshold established for normal blood flow) could then be established for different degrees of abnormal blood flow.

\section{COULD POST-TEST PROBABILITY OF DISEASE BE MORE USEFUL THAN SURVIVAL ANALYSIS?}

Post-test probability of disease can be assessed using likelihood ratios or negative predictive values. Such analyses consider the possibility that a negative MPI could be either a true negative or false negative. If the MPI is normal, a patient with a high pretest probability would still have an increased post-test probability of obstructive disease using typical negative likelihood ratios. This would not however inform clinicians with regard to disease free survival time in the same manner as a survival analysis.

Relative risk could be calculated after a normal MPI for individual patients according to their clinical risk profile. Hachamovitch et al. that demonstrated the relative risk for cardiac death or non-fatal MI following 

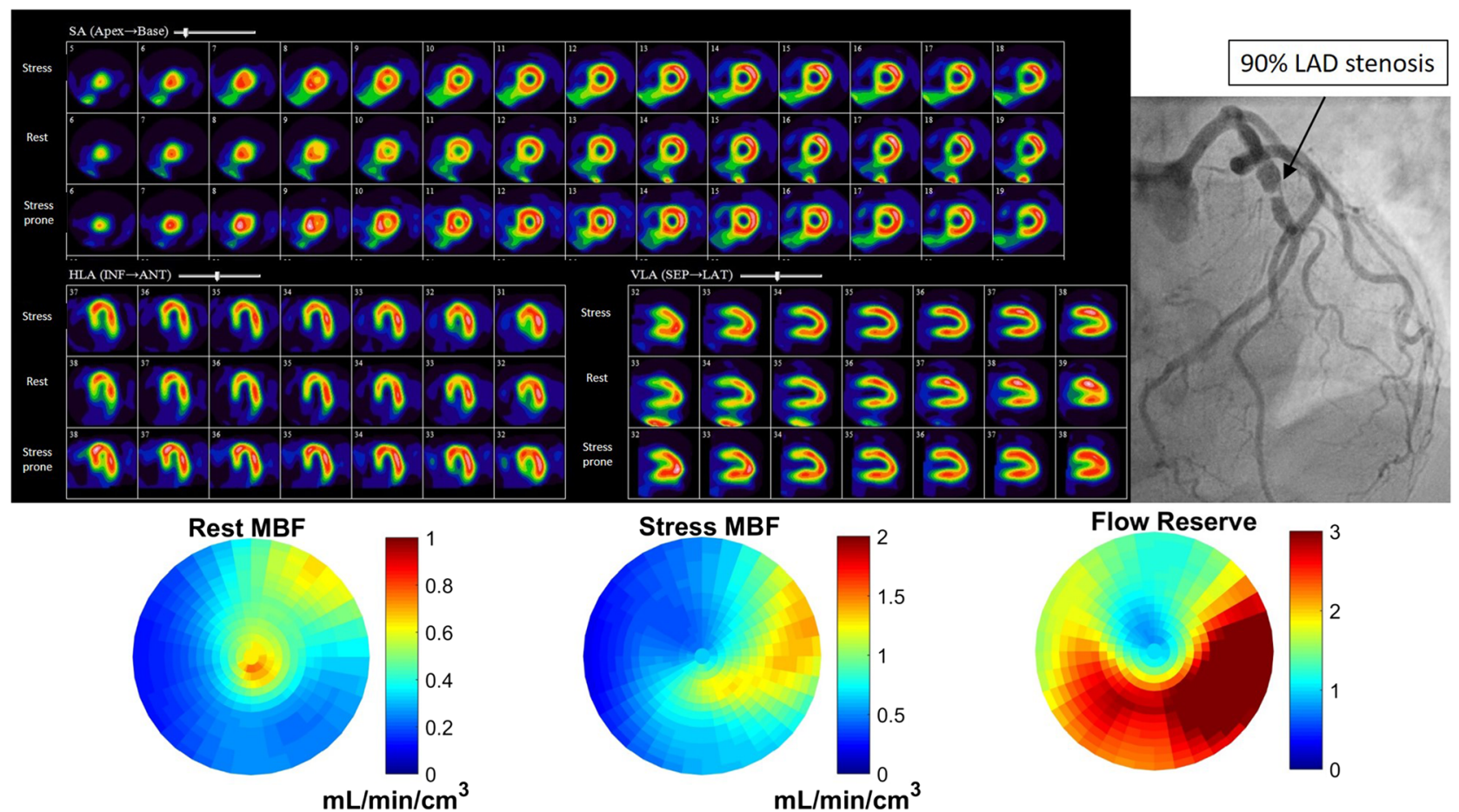

Figure 2. 55 year old male underwent exercise stress (top rows), rest (middle rows) and stress prone ${ }^{99 \mathrm{~m}}$ Tc-SPECT MPI with SPECT myocardial blood flow imaging. Relative perfusion images demonstrated an attenuation artifact on stress and rest images in the septum which partially resolves with prone imaging. There was normal anterior uptake on rest and stress images. Blood flow imaging demonstrated mildly reduced stress flow in the LAD territory and decreased flow reserve in these segments. A subsequent invasive coronary angiogram 2 weeks later demonstrated a severe (90\%) stenosis in the mid LAD and a successful percutaneous coronary intervention with a drug eluting stent was performed.

a normal MPI after 5 years of follow up was increased by 4.40 fold in diabetic versus non-diabetics (absolute event rate was $0.3 \%$ versus $1.2 \%$ for non-diabetics and diabetic respectively). ${ }^{2}$ The relative risk increase does not however indicate when the events are most likely to occur (early, late, or at a constant rate) and does not indicate whether there is an initial period of low risk following the MPI study.

\section{WARRANTY PERIOD IMPLICATIONS}

A clinical implication from warranty period studies is that not all patients with normal MPI studies can be managed in the same manner. Older patients, those with diabetes or hypertension or prior CAD will require closer surveillance than patients without these clinical risks. Surveillance might include closer attention to address cardiovascular risks such as diabetic control, achieving targets in blood pressure or lipid management, and referral to smoking cessation services. It might also advocate healthier lifestyles with regular exercise and adoption of a Mediterranean diet. Surveillance might also include more frequent testing once the warranty period had expired or might also lead to further personalization of risk stratification with a CT-CAC or CT angiogram in appropriate patients. Whether more rigorous attention to achieve risk factor targets or more frequent/ comprehensive testing might lengthen the warranty will require further clinical studies.

\section{CONCLUSIONS: FORECASTING THE FUTURE BASED ON NORMALCY AND RISK}

Acampa et al. demonstrate that hypertension and the type of stressor are important determinants of long term prognosis following a normal MPI. By using the established concept of a warranty period, the authors remind clinicians to be mindful that, even with a normal SPECT-MPI, patients with atherosclerotic risk factors remain susceptible to cardiac events in the long term. The term "warranty period", although not perfect for there are no guarantees or refunds for those who experience events, is a useful one which can be communicated easily with patients. It is perhaps more 
reassuring than using a term that incorporates uncertainty such as relative risk or hazard ratio.

The notion of a warranty period should not mislead clinicians as these patients may not be at low risk. Rather than terms such as "survival analysis" or "warranty period", the technique of combining atherosclerotic risks and normal MPI might be considered "forecasting". Our experiences with weather forecasting will help to achieve a clinical recognition that there is always a degree of uncertainty when planning ahead. In hypertensive patients with a normal MPI, the sun may be shining in the initial post scan period, but with clouds or risk factors on the horizon it may rain in the not too distant future.

\section{Disclosures}

Dr. Terrence D. Ruddy receives grant support from GE Healthcare and Advanced Accelerator Applications International and speaking honoraria from GE Healthcare. Dr. Gary $R$. Small has nothing to disclose.

\section{References}

1. Acampa W, Rozza F, Zampella E, Assante R, Mannarino T, Nappi $\mathrm{C}$, et al. Warranty period of normal stress myocardial perfusion imaging in hypertensive patients: A parametric survival analysis. J Nucl Cardiol. 2018. https://doi.org/10.1007/s12350-018-1285-9.

2. Hachamovitch R, Hayes S, Friedman JD, Cohen I, Shaw LJ, Germano G, et al. Determinants of risk and its temporal variation in patients with normal stress myocardial perfusion scans: what is the warranty period of a normal scan? J Am Coll Cardiol. 2003;41(8):1329-40.

3. Acampa W, Petretta M, Cuocolo R, Daniele S, Cantoni V, Cuocolo A. Warranty period of normal stress myocardial perfusion imaging in diabetic patients: a propensity score analysis. J Nucl Cardiol. 2014;21(1):50-6.

4. Romero-Farina G, Candell-Riera J, Aguade-Bruix S, FerreiraGonzalez I, Cuberas-Borros G, Pizzi N, et al. Warranty periods for normal myocardial perfusion stress SPECT. J Nucl Cardiol. 2015;22(1):44-54.

5. Kronmal RA, McClelland RL, Detrano R, Shea S, Lima JA, Cushman M, et al. Risk factors for the progression of coronary artery calcification in asymptomatic subjects: results from the Multi-Ethnic Study of Atherosclerosis (MESA). Circulation. 2007;115(21):2722-30.

6. Min JK, Lin FY, Gidseg DS, Weinsaft JW, Berman DS, Shaw LJ, et al. Determinants of coronary calcium conversion among patients with a normal coronary calcium scan: what is the "warranty period" for remaining normal? J Am Coll Cardiol. 2010;55(11):1110-7.

7. Hecht HS. Coronary artery calcium scanning: past, present, and future. JACC Cardiovasc Imaging. 2015;8(5):579-96.

8. Einstein AJ, Johnson LL, Bokhari S, Son J, Thompson RC, Bateman TM, et al. Agreement of visual estimation of coronary artery calcium from low-dose CT attenuation correction scans in hybrid PET/CT and SPECT/CT with standard Agatston score. J Am Coll Cardiol. 2010;56(23):1914-21.

9. Ghadri JR, Fiechter M, Fuchs TA, Scherrer A, Stehli J, Gebhard C, et al. Registry for the Evaluation of the PROgnostic value of a novel integrated imaging approach combining Single Photon Emission Computed Tomography with coronary calcification imaging (REPROSPECT). Eur Heart $\mathrm{J}$ Cardiovasc Imaging. 2013;14(4):374-80.

10. Klein R, Hung GU, Wu TC, Huang WS, Li D, deKemp RA, et al. Feasibility and operator variability of myocardial blood flow and reserve measurements with (9)(9)mTc-sestamibi quantitative dynamic SPECT/CT imaging. J Nucl Cardiol. 2014;21(6):107588.

11. Wells RG, Marvin B, Poirier M, Renaud J, deKemp RA, Ruddy TD. Optimization of SPECT Measurement of Myocardial Blood Flow with Corrections for Attenuation, Motion, and Blood Binding Compared with PET. J Nucl Med. 2017;58(12):2013-9.

12. Johnson NP, Gould KL. Integrating noninvasive absolute flow, coronary flow reserve, and ischemic thresholds into a comprehensive map of physiological severity. JACC Cardiovasc Imaging. 2012;5(4):430-40.

13. Ziadi MC, deKemp RA, Williams KA, Guo A, Chow BJ, Renaud $\mathrm{JM}$, et al. Impaired myocardial flow reserve on rubidium- 82 positron emission tomography imaging predicts adverse outcomes in patients assessed for myocardial ischemia. J Am Coll Cardiol. 2011;58(7):740-8. 